\title{
Electron Ion Collider: 3D-Imaging the Nucleon
}

\author{
Marco Radici ${ }^{1, a}$ \\ ${ }^{1}$ INFN - Sezione di Pavia, via Bassi 6 - 127100 Pavia - Italy
}

\begin{abstract}
The Electron Ion Collider (EIC) is the project for a new US-based, high-energy, high-luminosity facility, capable of a versatile range of beam energies, polarizations, and ion species. Its primary goal is to precisely image quarks and gluons and their interactions inside hadrons, in order to investigate their confined dynamics and elucidate how visible matter is made at its most fundamental level. I will introduce the main physics questions addressed by such a facility, and give some more details on the topic of Transverse Momentum Dependent parton distributions (TMDs).
\end{abstract}

\section{Introduction}

The Electron Ion Collider (EIC) is a US-based project to build the world's first electron-nucleus collider and the world's first electron-proton collider with both polarized electron and proton beams. The machine parameters were discussed in detail in the EIC White Paper [1, 2], and reviewed in the 2015 Nuclear Science Advisory Committee (NSAC) Long Range Plan where for the first time this project was established to be a priority for the entire nuclear physics community. They can be summarized by stating that the EIC is a huge high-resolution microscope with the following features:

- highly polarized (70\%) beams of electrons, protons and light nuclei,

- ion beams from deuteron to the heaviest nuclei (lead),

- a variable energy range in the center-of-mass $(\mathrm{cm})$ of the collision between $\sqrt{s} \sim 20$ to $100 \mathrm{GeV}$, upgradable to $\sim 140 \mathrm{GeV}$,

- collisions at very high luminosity $\sim 10^{33-34} \mathrm{~cm}^{-2} \mathrm{~s}^{-1}$,

- possibility of more than one interaction region.

Electron beams are needed to count on the unmatched precision of the electromagnetic interaction as a probe. High-energy and high-luminosity collisions are needed to construct a three-dimensional imaging of the internal structure of nucleons and nuclei in terms of distributions of quarks and gluons (collectively, partons) and their correlations. Polarization allows to explore the correlation of these parton distributions with the nucleon spin, and the separate contribution of quarks and gluons to the nucleon-nucleon interaction. Energy variability provides the tool to explore the transition from seaquark to gluon dominance, particularly in the nuclear environment. Finally, having a large set of available ion beams opens the way to systematically study how this nuclear environment influences the behaviour of partons, particularly of gluons at very large densities.

ae-mail: marco.radici@pv.infn.it 
The EIC science case is thus broad and diverse, but it can be summarized as the attempt of understanding how the structure of the visible matter in the universe comes about in the framework of the Standard Model theory of strong interactions, the Quantum Chromodynamics (QCD). In QCD, the quarks and gluons interact because they have a color charge. At variance with electromagnetism, gluons carry color charge and can interact with each other. Hence, QCD is an intrinsically nonlinear theory. While at very small separations quarks and gluons interact weakly and perturbative techniques can be applied to solve QCD with spectacular results (see, for example, Ref. [3] and references therein), the visible hadronic matter is formed by quark-gluon interactions at the typical scale of the proton size, namely of one or few Fermi. At such separations, the quark-gluon force is so large that QCD cannot be calculated from first principles. Neither lattice QCD (which tries to solve the nonabelian gauge theory on a discretized space-time lattice) is the ultimate answer: parton distributions are studied in the socalled light-cone kinematics, and lattice simulations cannot presently address this dynamical regime. The most intriguing puzzle of the nonlinear QCD is color confinement, namely the fact that free quarks and gluons (free color charges, in general) cannot be observed: partons are permanently confined inside color-neutral hadrons. This evidence hampers any attempt of elucidating the microscopic details of such nonlinear dynamics. As a consequence, several questions are still open that have large impact also on other fields in physics. The EIC project aims to address them in detail.

\section{Open questions in QCD}

Perhaps the most relevant problem is understanding the origin of the nucleon mass. We know that in the Standard Model the Higgs mechanism provides the mass of quarks, that are external parameters to the QCD Lagrangian. For valence quarks (i.e., those which contribute to the quantum numbers of the nucleon), these masses are nearly one hundredth smaller than that of the nucleon. Gluons carry no mass. It is only thanks to the strong interaction of quarks and gluons with each other and with the QCD vacuum that the generated energy transforms into the observed mass of the proton and the neutron, the most common elements in the universe. The QCD dynamics of partonic fields is thus responsible of approximately $99 \%$ of the mass of all visible matter [4]. Not only, it is responsible also for the tiny difference between the proton and the neutron masses which is at the origin of the nucleosynthesis and the formation of stars. Why is all this a problem? Because we are not able to calculate this nonlinear dynamics at the scale of the proton size. Therefore, we cannot learn all details that bring confined interacting partons to eventually produce the observed hadron masses. The same argument applies also to other macroscopic properties of the nucleon like its size (for a review, see Ref. [8]) and spin.

Since the first measurement of the EMC collaboration [10], we know that only a small fraction ( $\lesssim 30 \%$ ) of the proton spin is carried by quarks. Only recently, we got the experimental evidence that the gluon gives a nonvanishing contribution [11]. But the still large uncertainty in both the experimental measurements and in the theoretical analysis makes it difficult to conclude that this contribution does or does not saturate the proton spin sum rule. This means that in principle there is room for other contributions coming from the orbital motion of partons inside the proton [12, 13]. Even if the sum of these contributions from different flavors would compensate and give a vanishing share of the proton spin, still it would be interesting to study how the cancellation would come about. At the quantitative level, we have only speculative calculations of the nucleon matrix elements of the partonic orbital angular momentum operator either in models (see, e.g., Ref. [14]) or in lattice QCD [15]. In fact, the definition of this operator is in general not unique in an interacting quantum field theory like QCD [16]. An unambiguous formulation implies the use of new partonic correlation 
operators, the Wigner distributions of quarks and gluons [17], whose extraction from experimental data is still under debate [18].

The nonlinearity of QCD is responsible also for a proliferation of the number of gluons when we probe a hadronic target at very large $\mathrm{cm}$ energies $\sqrt{s}$ (or, equivalently, at very small fractional momenta $x$ carried by partons). In fact, a colored gluon can split into two colored gluons. At small $x$, the splitting is favored and the net result is a dramatic increase of the gluon density distribution. But this rising cannot continue forever because it would violate the unitarity in the cross section. When the density reaches some critical value, pairs of gluons must start to recombine into a single gluon at a rate that balances the splitting. This phenomenon is called saturation [19]. Where does it set in? The idea is that there should be a threshold scale depending on $x$, named saturation scale $Q_{s}(x)$, that separates the condensed and saturated gluonic matter from the dilute and confined quarks and gluons. However, nobody has ever seen evidence of this new gluonic matter in the experimental data so far. Does $Q_{s}(x)$ depend on the chosen hadron target or is it universal? In other words, is there a universal gluonic matter (often referred to as the Color Glass Condensate - CGC - a new state of matter [19]) at very large densities? More generally, how does nuclear matter affect the quark-gluon interaction?

The possibility of using heavy ion beams at EIC could provide a precocious access to saturation because the virtual photon would probe matter coherently over a length proportional to $1 / x$, which can exceed the diameter of a Lorentz-contracted nucleus. Then, all gluons at the same impact parameter would contribute to the probed density, reaching saturation at lower energies than with a electronproton collisions. Therefore, the EIC could be the first machine to provide experimental evidence of saturated gluonic matter and to validate the CGC hypothesis. At the same time, with collisions at high luminosity and with a high degree of polarization it will be possible to build a three-dimensional picture of the internal motion of partons both in space and momentum. This sort of nucleon tomography should elucidate many aspects of the nonlinear dynamics of gluons and of different flavors of quarks, with particular emphasys on the spin-momentum correlations contributing to the proton spin sum rule. In this respect, the combination of high luminosity and wide reach in cm energy with high polarization in both colliding beams, and with a large selection of ion beams, makes the EIC a unique facility in the panorama of electron scattering machines of both fixed-target and collider types [2].

In the following, we focus on the new perspective of building a tomography of the nucleon. In particular, we consider the recent achievements in momentum space using new tools named Transversemomentum dependent parton distributions (TMDs).

\section{Nucleon tomography}

There are different starting points to explore the internal structure of the nucleon. Spectroscopy is the doorway to explore QCD at work when combining quarks and gluons to form bound states, including unexpected exotic configurations like glueballs, tetraquarks and pentaquarks. Alternatively, elastic scattering with large $\mathrm{cm}$ energies allows to catch inner details of the hadron structure. The typical adopted regime is called Deep-Inelastic Scattering (DIS), where the space-like 4-momentum $q$ transferred to the target becomes larger and larger while keeping the ratio $x_{B}=Q^{2} / 2 P \cdot q$ fixed, with $P$ the target 4-momentum and $Q^{2}=-q^{2} \geq 0$. The kinematics is highly relativistic and it is useful to describe the system in terms of Light-Cone (LC) variables (a 4-vector $a$ is represented as $a=\left\{a^{+}, a^{-}, \boldsymbol{a}_{T}\right\}$, with $\left.a^{ \pm}=\left(a^{0} \pm a_{z}\right) / \sqrt{2}\right)$. In fact, if the target is initially at rest, after absorbing the virtual photon it gets boosted along the direction of the spatial momentum transfer, say $\hat{z}$. Since $Q \rightarrow \infty$, the target momentum has a dominant component, $P^{+} \sim Q$, while $P^{-} \sim 1 / P^{+}$is suppressed and can be neglected. The target gets squeezed as a disk along the boost direction by Lorentz contraction, and it can be approximated as an assembly of partons traveling collinearly with the parent hadron, with "LC-longitudinal" 
momenta $p^{+}=x P^{+}$and negligible transverse momenta $\left|\boldsymbol{k}_{T}\right| \ll p^{+}$, with $x \sim x_{B}$. This highly relativistic framework, conventionally named Infinite Momentum Frame (IMF), is the framework where ordinary Parton Distribution Functions (PDFs) are defined and extracted from data as functions of the partonic fractional momentum $x$ and of the hard scale $Q^{2}$.

However, in the previous section we have already come across an experimental evidence that this picture is not exhaustive: the possibility that "spin-orbit" correlations can have a nonvanishing share of the proton's spin points out the dynamical relevance of the transverse momentum $\boldsymbol{k}_{T}$ of partons with respect to the parent hadron momentum. Therefore, we need to go beyond the simple monodimensional picture where details of parton dynamics come only from the PDFs as functions of the collinear partonic momentum fraction $x$.

In principle, the maximum information available about the dynamics of partons moving inside the nucleon in the IMF is contained in the socalled Wigner distributions $W_{S, S_{q}}\left(x, \boldsymbol{k}_{T}, \boldsymbol{b}\right)$, which describe the correlation between the transverse momentum $\boldsymbol{k}_{T}$ and position $\boldsymbol{b}$ of a parton with helicity $S_{q}$ and "LC-longitudinal" fraction $x$ of the momentum of the parent nucleon with helicity $S$ [20]. The transverse distance $\boldsymbol{b}$ (or impact parameter) is measured relative to the center of mass $\boldsymbol{R}$ in the transverse plane, which can be localized to zero by constructing a superposition of nucleon states with transverse momenta $\boldsymbol{P}_{T} \ll P^{+}$(in order to comply with the IMF interpretation of the nucleon as an assembly of many collinear partons) [21].

By construction, the 5-dimensional $W$ is not positive-definite, i.e. it is not a probability density. But it is not constrained by the Heisenberg principle because $\boldsymbol{b}$ is not the conjugate variable of $\boldsymbol{k}_{T}$ but rather of the variation of transverse nucleon momentum in the scattering process, namely $\boldsymbol{q}=\boldsymbol{P}_{T}^{\prime}-\boldsymbol{P}_{T}$ [22]. As such, the Wigner distributions are defined as matrix elements between different nucleon states of various nonlocal quark and gluon operators, depending on the parton and/or nucleon polarization state. At present, it is not clear whether there is a process where the Wigner distributions could be extracted from; suggestions have recently appeared in the literature [18] and are being scrutinized. The quark orbital angular momentum (OAM) operator can be defined starting from the Wigner distribution for an unpolarized quark $q$ in a longitudinally polarized nucleon [17],

$$
l_{z}^{q}=\int d \boldsymbol{b}\left[\boldsymbol{b} \times \int d x d \boldsymbol{k}_{T} \boldsymbol{k}_{T} W_{L U}^{q}\left(x, \boldsymbol{k}_{T}, \boldsymbol{b}\right)\right]_{z}=\int d \boldsymbol{b}\left[\boldsymbol{b} \times\left\langle\boldsymbol{k}_{T}^{q}\right\rangle(\boldsymbol{b})\right]_{z},
$$

where $\left\langle\boldsymbol{k}_{T}^{q}\right\rangle(\boldsymbol{b})$ is the average transverse momentum carried by a quark $q$ at a certain transverse position $\boldsymbol{b}$. The OAM is then built by generalizing its classical definition, and the $l_{z}^{q}$ operator satisfies the canonical commutation relations.

By integrating the $\boldsymbol{k}_{T}$-dependence of the Wigner distributions $W$, we can build new partonic densities in configuration space, namely the impact-parameter distributions $q(x, \boldsymbol{b})$ [22]. They describe the probability density for a parton with flavor $q$ and "LC-longitudinal" momentum $x$ to be located in the transverse plane at distance $\boldsymbol{b}$ from the transverse center-of-mass $\boldsymbol{R}=0$. Hence, for each $x$ we can build a snapshot of the nucleon in the transverse plane: we can make a nucleon tomography.

By integrating the $\boldsymbol{b}$ dependence of the Wigner distributions $W$, we can build new partonic densities in momentum space, the Transverse-momentum dependent parton distributions $q\left(x, \boldsymbol{k}_{T}\right)$ (TMDs). They describe the probability density of finding a parton with flavor $q$ (and polarization $S_{q}$ ) carrying a "LC-longitudinal" momentum $x$ and a transverse momentum $\boldsymbol{k}_{T}$ at the hard scale $Q^{2}$. We stress that the TMDs $q\left(x, \boldsymbol{k}_{T}\right)$ are not simply the Fourier transform of the impact-parameter distributions $q(x, \boldsymbol{b})$ because $\boldsymbol{k}_{T}$ is not conjugated to $\boldsymbol{b}$. Hence, the information delivered by TMDs is indeed independent and complementary. In the following, we summarize our current knowledge about TMDs. 


\section{Transverse momentum distributions}

In the DIS regime, the TMDs emerge as leading coefficients in the expansion of the scattering amplitude in $1 / Q^{2}$ (leading twist). For a spin- $\frac{1}{2}$ hadron (e.g., the proton), there are eight TMDs at leading twist. They are grouped according to the unpolarized (U), longitudinal $(\mathrm{L})$, or transverse $(\mathrm{T})$ polarization state of the parton or of the proton. By integrating their $\boldsymbol{k}_{T}$ dependence, we recover the three independent PDFs (as functions of $x$ ) that fully describe the collinear partonic structure of the nucleon at leading twist: the well known unpolarized momentum distribution $f_{1}$ and helicity distribution $g_{1}$, and the less known transversity distribution $h_{1}$, which has been extracted only recently by two independent procedures [23-25]. The combinations corresponding to longitudinally polarized quarks in unpolarized protons (LU) and viceversa (UL) are forbidden by imposing the invariance of the scattering amplitude under parity transformations. The TMDs involving transversely polarized quarks are named chiral-odd because they are connected to processes where the quark helicity is flipped (at leading twist, helicity and chirality are the same). As such, the chiral-odd TMDs should be suppressed in QCD where helicity is conserved up to terms proportional to (small) $m / Q$ (with $m$ the quark mass). Nevertheless, they appear in scattering amplitudes at leading twist and are responsible of visible and measurable effects.

The TMDs can be extracted only in processes with two scales: a hard one $\left(Q^{2}\right)$ that localizes the probe and enables "to see" the partons, and a soft one that is sensitive to the transverse momenta of partons confined at the scale of the proton size. The typical example is a Semi-Inclusive process in the DIS regime (SIDIS) where one measures the azimuthal angle of the final-state products around a reference axis defined in the lab frame. If the transverse momentum $\left|\boldsymbol{P}_{h T}\right|$ of the outgoing particles is much smaller than the hard scale $Q$ of the process, a specific factorization proof has been put forward that allows to isolate TMDs in the scattering amplitude [26, 27]. In this framework (called TMD framework), the SIDIS cross section can be parametrized in terms of various structure functions (depending on the polarization of the beam/target/final products), each one involving a specific TMD (and a corresponding fragmentation function for the final products) [28]. Each structure function has a peculiar dependence upon the azimuthal angle of the final products, as well as the azimuthal angle of the various polarization vectors involved. Consequently, it can be isolated by constructing specific azimuthal asymmetries, sometimes involving a spin flip of a polarized particle (in which case, we deal with Single-Spin Asymmetries - SSAs).

Several experimental measurements of SIDIS azimuthal and spin asymmetries were performed in the last 15 years, addressing each one of the eight TMDs (for a review of the current situation see Ref. [29]). However, the collected amount of data is not yet sufficient to grant a knowledge of TMDs comparable to what we have learnt so far about PDFs. Only recently, first attempts to parametrize the unpolarized $f_{1}^{q}\left(x, \boldsymbol{k}_{T}\right)$ have appeared in the literature $[30,31]$, in order to explore if and how the $\boldsymbol{k}_{T}$ dependence changes with flavor $q$ and with $x$. The $f_{1}^{q}\left(x, \boldsymbol{k}_{T}\right)$ occurs also in the leading-twist part of the cross section for unpolarized proton-proton collisions leading to a final pair of leptons (Drell-Yan process), if the transverse momentum $\boldsymbol{q}_{T}$ of the virtual decaying boson is much smaller than the invariant mass $Q$ of the lepton pair (the hard scale) [32]. Recent extractions of this TMD from these data have been released with very sophisticated analyses [33-35]. Interestingly, it emerges that the nonperturbative details of TMDs have a visible impact also on the low $\boldsymbol{k}_{T}$-tail of the transverse momentum distribution for $Z$ boson production at LHC [34], possibly affecting also some Standard Model parameters like the $W$ boson mass and, consequently, modifying some thresholds and/or sensitivities to effects from New Physics beyond that. By comparing the available analyses of both SIDIS and Drell-Yan processes, an explicit indication has been obtained that the average width $\left\langle\boldsymbol{k}_{T}^{2}\right\rangle$ depends on the cm energy available in the process [36]. 
But only recently the first attempt of extracting $f_{1}^{q}\left(x, \boldsymbol{k}_{T}\right)$ from a global fit has been finalized [37]. The analysis has been carried out in the kinematic limits $M^{2}, \boldsymbol{P}_{h T}^{2} \ll Q^{2}$ for SIDIS and $\boldsymbol{q}_{T}^{2} \ll Q^{2}$ for Drell-Yan, at leading order (LO) in the strong coupling constant $\alpha_{s}$ and resumming important QCD radiative corrections at the next-to-leading logarithmic level (NLL). Within these approximations, the SIDIS cross section is proportional to a convolution of $f_{1}^{q}\left(x, \boldsymbol{k}_{T}\right)$ and the function $D_{1}^{q \rightarrow h}\left(z, \boldsymbol{P}_{\perp}\right)$ for the fragmentation of a quark $q$ into a hadron $h$ carrying fractional energy $z$ and transverse momentum $\boldsymbol{P}_{\perp}$. The Drell-Yan cross section simplifies to a convolution of $f_{1}^{q}\left(x, \boldsymbol{k}_{T}\right)$ and $f_{1}^{\bar{q}}\left(x, \boldsymbol{k}_{T}\right)$ for the corresponding antiquark in the annihilation. The evolution of TMDs from a starting scale $Q_{0}$ to a final scale $Q$ is better studied in configuration space introducing the dependence on the conjugate variable $\xi$ [38]. In the LO and NLL approximation, it reads [37]

$$
f_{1}^{q}\left(x, \boldsymbol{\xi}^{2}, Q^{2}\right)=f_{1}^{q}\left(x, \mu_{b}^{2}\right) e^{S\left(\mu_{b}^{2}, Q^{2}\right)} e^{S_{\mathrm{NP}}\left(\xi^{2}\right) \log \left(Q^{2} / Q_{0}^{2}\right)} f_{1 \mathrm{NP}}^{q}\left(x, \xi^{2}\right) .
$$

According to the CSS formalism [38], the resummation of soft gluon radiation is contained in the exponential form of the Sudakov form factor $S$. The collinear PDF is evaluated at the scale $\mu_{b}$ which depends on $\xi^{2}$ such that it is always constrained between $Q_{0}$ and $Q$. When $Q=Q_{0}$, there are no evolution effects and the TMD falls back into the product of a collinear PDF at $\mu_{b} \equiv Q_{0}$ multiplied by a nonperturbative function of $\boldsymbol{\xi}$. The latter contains free parameters that need to be fixed to experimental data. It is made of two terms: the exponential form of a nonperturbative Sudakov form factor $S_{\mathrm{NP}}$ and the arbitrary function $f_{1 \mathrm{NP}}$. The former is usually chosen as $S_{\mathrm{NP}}=-g_{2} \xi^{2} / 2$ with $g_{2}$ a fitting parameter. In Ref. [37], the latter is chosen as the normalized linear combination of two Gaussians with the same width $g_{1}(x)$. An evolution equation similar to Eq. (2) holds also for the fragmentation function $D_{1}$, whose $D_{1 \mathrm{NP}}$ is parametrized as a linear combination of Gaussians with different widths $g_{3}(z), g_{4}(z)$. The coefficients of the two linear combinations are fitting parameters. Also $g_{1}(x)$ and $g_{3,4}(z)$ contain fitting parameters, for a total of 11 free parameters. Their best values are determined using the bootstrap method. A set of $\mathcal{M}$ replicas of the data points is created by altering them with a Gaussian noise with the same variance as the experimental measurement. For each replica $r$, the set of new points is fitted as if it were an independent experiment, thus producing a vector of parameters $V_{r}$. The number $\mathcal{M}$ is fixed by accurately reproducing the mean and standard deviation of the original data points. In the following plots, it is used $\mathcal{M}=200$ and the uncertainty band is constructed by taking the central $68 \%$ of the 200 replicas, namely by rejecting the largest and smallest $16 \%$ of them for each experimental bin [37]. If the 200 theoretical outcomes were Gaussian-distributed, the central $68 \%$ would correspond to the standard $1 \sigma$ confidence interval.

The SIDIS data have been taken by the HERMES [39] and COMPASS [40] collaborations using proton and deuteron targets and detecting various types of final hadrons. The HERMES data set covers the range $0.04<x<0.4$, while for COMPASS $0.005<x<0.12$. Several kinematical cuts were applied in order to ensure that the TMD factorization framework is applicable and that only the current fragmentation region is explored: $Q^{2}>1.4 \mathrm{GeV}^{2}, P_{h T}<\min [0.2 Q, 0.7 z Q]+0.5 \mathrm{GeV}$, and $0.2<z<0.74$. These cuts reduced the considered bins to 1514 and 6252 for HERMES and COMPASS, respectively. The results are displayed in Fig. 1. Each box corresponds to a specific pair of $\langle x\rangle,\left\langle Q^{2}\right\rangle$ bins. In each box, the various curves are the $P_{h T}$ distributions for different $\langle z\rangle$ bins, where each curve is shifted by an offset indicated in the legenda. Since the published COMPASS data are affected by normalization errors (see the erraturm to Ref. [40]), the theoretical results are normalized to the first $P_{h T}$ data point for each $\langle z\rangle$ bin. The bands are the result of the global fit in Ref. [37].

The Drell-Yan data were collected at the Tevatron in different times, and are here organized in a set with final lepton pairs at low invariant mass and another set at the $Z$ mass. The first data set was produced by the E288 [41] and E605 [42] collaborations. After imposing the cut $q_{T}<0.2 Q+0.5$ $\mathrm{GeV}$ to comply with the TMD factorization framework, the total amount of fitted bins reduces to 

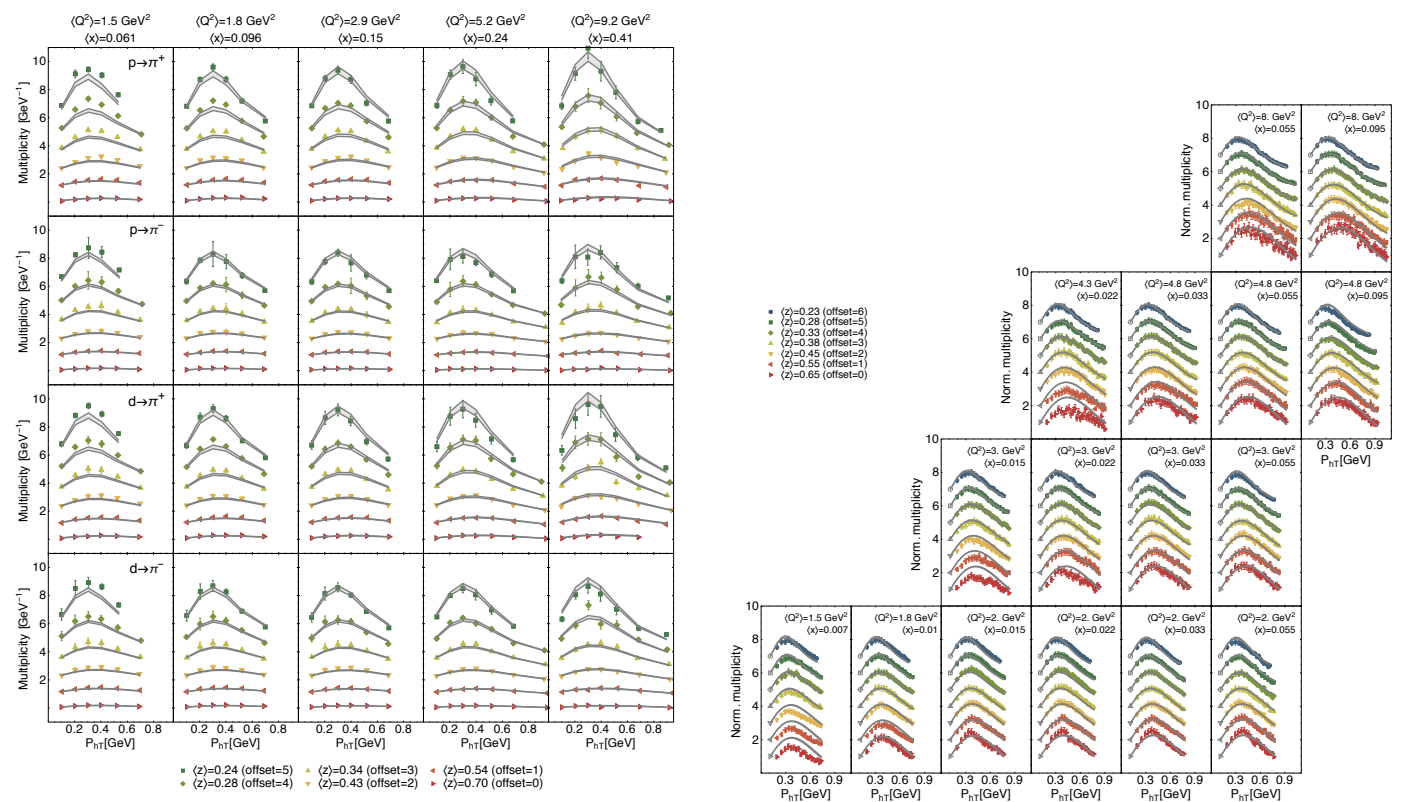

Figure 1. SIDIS multiplicities of charged pions. Left panel: results from the HERMES collaboration [39] for proton (upper two rows) and deuteron (lower two rows) targets. Right panel: results from the COMPASS collaboration [40] for negatively charged hadrons off deuteron targets. Each box corresponds to specific $\left(\langle x\rangle,\left\langle Q^{2}\right\rangle\right)$ bins. The curves are $P_{h T}$ distributions at different $\langle z\rangle$ bins, shifted by an offset specified in the legenda. The bands are the result of the global fit from Ref. [37].

203. In the right panel of Fig. 2, from left to right the first three plots refer to the E288 results for collisions of protons on $\mathrm{Cu}$ or Pt targets at three different $\mathrm{cm}$ energies $\sqrt{s}=19.4,23.8,27.4 \mathrm{GeV}$. In each plot, the curves are the $q_{T}$ distributions at different values of the invariant mass $\langle Q\rangle$ of the produced $\mu^{+} \mu^{-}$pair. The rightmost plot contains the E605 results in the same notations for collisions of protons on a copper fixed target at $\sqrt{s}=38.8 \mathrm{GeV}$. For clarity, each $\langle Q\rangle$ bin has been normalized to the first data point and then shifted by an offset indicated in the legend. In the right panel, the data points collected by the CDF and DQ collaborations at the Tevatron are shown for two different runs at $\sqrt{s}=1.8 \mathrm{TeV}[43,45]$ and $\sqrt{s}=1.96 \mathrm{TeV}[44,46]$, where a proton and an antiproton collide producing $e^{+} e^{-}$pairs with invariant mass $Q \approx M_{Z}$. The same previous kinematical cut is applied on $q_{T}$ so that the amount of fitted bins is 90 . The theoretical curves are the result of the global fit from Ref. [37], and are normalized according to the factors listed in Ref. [34] and obtained by comparing the experimental total cross section with the one computed in Ref. [47].

In summary, the global fit of Ref. [37] considered a set of 8059 data points using a vector $V$ of 11 free parameters for a total $\chi^{2}$ per degree of freedom equal to $1.55 \pm 0.05$. Using the replica method, the global fit produces $V_{r}, r=1, . ., 200$, parameter vectors, from which we can construct any related observable. In particular, the average transverse momentum $\left\langle\boldsymbol{k}_{\perp}^{2}\right\rangle(x)$ of the TMD in Eq. (2) and of the related fragmentation function $\left\langle\boldsymbol{P}_{\perp}^{2}\right\rangle(z)$ can be analytically computed and are shown in Fig. 3 . We recall that the uncertainty bands are formed from the central $68 \%$ of the 200 replicas of the average values. These bands are the first example of hadron tomography in momentum space because they 

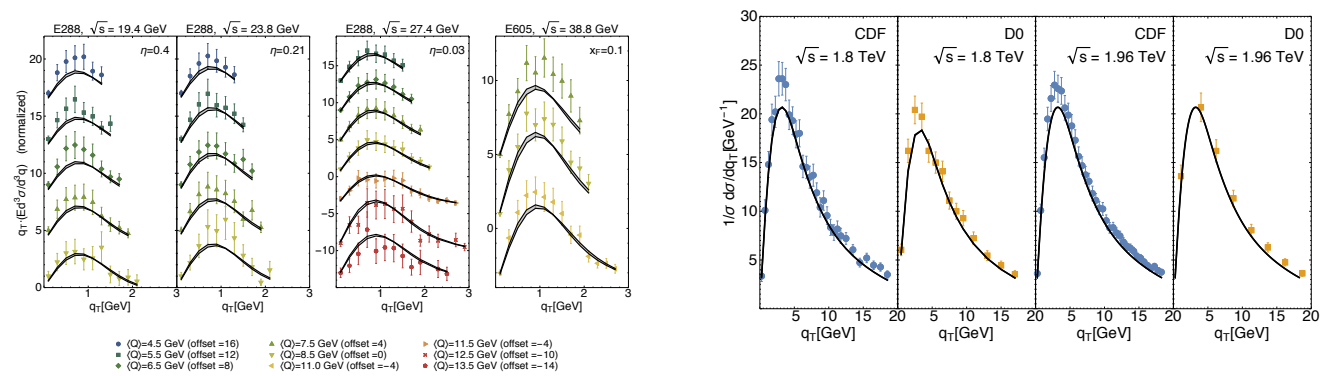

Figure 2. The Tevatron data for the Drell-Yan cross section. Left panel: results from the E288 collaboration [41] at $\mathrm{cm}$ energies $\sqrt{s}=19.4,23.8,27.4 \mathrm{GeV}$ (three leftmost plots) and from the E605 collaboration [42] at $\sqrt{s}=$ 38.8. GeV. Right panel: results from Run I and II at $\sqrt{s}=1.8$ and $1.96 \mathrm{TeV}$ from the CDF $[43,44]$ and DQ $[45,46]$ collaborations, respectively. The curves are $q_{T}$ distributions of the final lepton pair at different invariant masses $\langle Q\rangle$, shifted by an offset as indicated in the legenda. In the right panel, the invariant mass is $Q \approx M_{Z}$. The bands are the result of the global fit from Ref. [37].
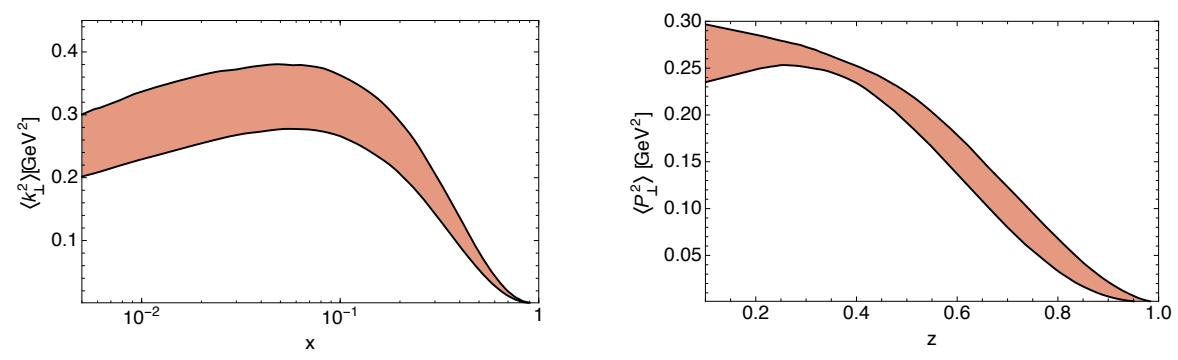

Figure 3. In the left panel, the average transverse momentum squared $\left\langle k_{\perp}^{2}\right\rangle$ of a quark confined in a proton as a function of its longitudinal momentum fraction $x$. In the right panel, the same for the fragmenting quark with $P_{\perp}$ as a function of the fractional energy $z$ of the detected hadron. The bands are the envelope at $68 \%$ confidence level of the full set of best-fit curves [37]. The data used in the global fit approximately cover the range $0.005 \lesssim x \lesssim 0.5$ and $0.2 \lesssim z \lesssim 0.7$.

show at $68 \%$ confidence level a snapshot of the average transverse momentum distribution of partons for each different longitudinal fractional momentum (and similarly for the fragmenting parton).

\section{Outlooks}

Several improvements upon the theoretical approach used in the global fit discussed in the previous section, should be explored in order to get more realistic results. In short, a flavor dependence should be introduced in the fitting parameters to reflect the natural expectation that transverse momentum distributions of different flavors should be different. The analysis is based on the assumption that the functional form of TMDs is a linear combination of Gaussians: other forms should be explored, also for the arbitrary nonperturbative part of the evolution operator. A more refined analysis of the perturbative calculable part of TMDs should be attacked which would probably allow for a better 
description of data at larger transverse momenta, and would relax the tension in the normalization of theoretical results to data points.

More experimental data are also needed for several reasons. The analysis of SIDIS data typically returns a strong anticorrelation between the average tranverse momenta of TMD and of the corresponding fragmentation functions $[30,37]$. The discussed results show that including the Drell-Yan data into the fit smooths this anticorrelation but it does not solve the problem. Only $e^{+} e^{-}$data for transverse momentum dependent fragmentation functions could provide the independent information that would overcome anticorrelation. But these data are still missing. In general, more data are needed to constrain the arbitrary nonperturbative part of TMD evolution [48]. From this point of view, a large range in $Q^{2}$ should be spanned.

The discussed results concern quark TMDs. At present, almost nothing is known about gluon TMDs because they typically require higher-energy scattering processes. Several promising measurements have been proposed which include isolated photon-pair production at RHIC [49] and quarkonium production at LHC [50-52]. But the cleanest possibility perhaps would be to look for dijet and heavy quark pair production in electron-proton collisions at the future EIC [53, 54]. The available larger phase space in $x, Q^{2}, P_{h T}, z$, really makes the EIC project a crucial step in the global determination of TMD and, more generally, in the 3-dimensional exploration of the internal structure of hadrons in both configuration and momentum space.

\section{References}

[1] D. Boer, M. Diehl, R. Milner, R. Venugopalan, W. Vogelsang et al. (2011), 1108. 1713

[2] A. Accardi et al., Eur. Phys. J. A52, 268 (2016), 1212.1701

[3] A. De Roeck, R.S. Thorne, Prog. Part. Nucl. Phys. 66, 727 (2011), 1103.0555

[4] B. Mueller, Nucl. Phys. A 750, 84 (2005)

[5] R. Pohl, A. Antognini, F. Nez, F.D. Amaro, F. Biraben et al., Nature 466, 213 (2010)

[6] P.J. Mohr, B.N. Taylor, D.B. Newell, Rev. Mod. Phys. 84, 1527 (2012), 1203.5425

[7] J.C. Bernauer et al. (A1), Phys. Rev. Lett. 105, 242001 (2010), 1007. 5076

[8] R. Pohl, R. Gilman, G.A. Miller, K. Pachucki, Ann. Rev. Nucl. Part. Sci. 63, 175 (2013), 1301.0905

[9] V. Punjabi, C.F. Perdrisat, M.K. Jones, E.J. Brash, C.E. Carlson, Eur. Phys. J. A51, 79 (2015), 1503.01452

[10] J. Ashman et al. (European Muon), Phys. Lett. B206, 364 (1988)

[11] D. de Florian, R. Sassot, M. Stratmann, W. Vogelsang, Phys. Rev. Lett. 113, 012001 (2014), 1404.4293

[12] R.L. Jaffe, A. Manohar, Nucl. Phys. B337, 509 (1990)

[13] X. Ji, X. Xiong, F. Yuan, Phys. Rev. Lett. 109, 152005 (2012), 1202.2843

[14] C. Lorce, B. Pasquini, X. Xiong, F. Yuan, Phys.Rev. D85, 114006 (2012), 1111.4827

[15] M. Deka et al., Phys. Rev. D91, 014505 (2015), 1312.4816

[16] E. Leader, C. Lorce, Phys.Rept. (2013), 1309. 4235

[17] C. Lorcé, B. Pasquini, Phys.Rev. D84, 014015 (2011), 1106.0139

[18] S. Bhattacharya, A. Metz, J. Zhou, Phys. Lett. B771, 396 (2017), 1702 . 04387

[19] E. Iancu, A. Leonidov, L.D. McLerran, Nucl. Phys. A692, 583 (2001), hep-ph/0011241

[20] C. Lorce, B. Pasquini, M. Vanderhaeghen, JHEP 1105, 041 (2011), 1102 . 4704

[21] G.A. Miller, Phys. Rev. Lett. 99, 112001 (2007), 0705 . 2409

[22] M. Burkardt, Int.J.Mod.Phys. A18, 173 (2003), hep-ph/0207047 
[23] M. Anselmino, M. Boglione, U. D’Alesio, S. Melis, F. Murgia et al., Phys.Rev. D87, 094019 (2013), 1303.3822

[24] Z.B. Kang, A. Prokudin, P. Sun, F. Yuan, Phys. Rev. D93, 014009 (2016), 1505.05589

[25] M. Radici, A. Courtoy, A. Bacchetta, M. Guagnelli, JHEP 05, 123 (2015), 1503.03495

[26] J.C. Collins, A. Metz, Phys. Rev. Lett. 93, 252001 (2004), hep-ph/0408249

[27] X. Ji, J.P. Ma, F. Yuan, Phys. Lett. B597, 299 (2004), hep-ph/0405085

[28] A. Bacchetta, M. Diehl, K. Goeke, A. Metz, P.J. Mulders, M. Schlegel, JHEP 02, 093 (2007), hep-ph/0611265

[29] H. Avakian, A. Bressan, M. Contalbrigo, Eur. Phys. J. A52, 150 (2016), [Erratum: Eur. Phys. J.A52,no.6,165(2016)]

[30] A. Signori, A. Bacchetta, M. Radici, G. Schnell, JHEP 1311, 194 (2013), 1309. 3507

[31] M. Anselmino, M. Boglione, J.O.G. H., S. Melis, A. Prokudin (2013), 1312.6261

[32] J.C. Collins, D.E. Soper, G. Sterman, Nucl. Phys. B250, 199 (1985)

[33] A.V. Konychev, P.M. Nadolsky, Phys. Lett. B633, 710 (2006), hep-ph/0506225

[34] U. D’Alesio, M.G. Echevarria, S. Melis, I. Scimemi, JHEP 11, 098 (2014), 1407. 3311

[35] I. Scimemi, A. Vladimirov (2017), 1706.01473

[36] P. Schweitzer, T. Teckentrup, A. Metz, Phys. Rev. D81, 094019 (2010), 1003.2190

[37] A. Bacchetta, F. Delcarro, C. Pisano, M. Radici, A. Signori, JHEP 06, 081 (2017), 1703. 10157

[38] J. Collins, Foundations of Perturbative QCD, Cambridge Monographs on Particle Physics, Nuclear Physics and Cosmology (Cambridge University Press, 2011), ISBN 9780521855334, http://books.google.it/books?id=0xGi1KW9vykC

[39] A. Airapetian et al. (HERMES Collaboration), Phys. Rev. D87, 074029 (2013), 1212 . 5407

[40] C. Adolph et al. (COMPASS), Eur. Phys. J. C73, 2531 (2013), [Erratum: Eur. Phys. J.C75,no.2,94(2015)], 1305.7317

[41] A.S. Ito et al., Phys. Rev. D23, 604 (1981)

[42] G. Moreno et al., Phys. Rev. D43, 2815 (1991)

[43] T. Affolder et al. (CDF), Phys. Rev. Lett. 84, 845 (2000), hep-ex/0001021

[44] T. Aaltonen et al. (CDF), Phys. Rev. D86, 052010 (2012), 1207.7138

[45] B. Abbott et al. (D0), Phys. Rev. D61, 032004 (2000), hep-ex/9907009

[46] V.M. Abazov et al. (D0), Phys. Rev. Lett. 100, 102002 (2008), 0712.0803

[47] S. Catani, L. Cieri, G. Ferrera, D. de Florian, M. Grazzini, Phys. Rev. Lett. 103, 082001 (2009), 0903.2120

[48] A. Bacchetta, M.G. Echevarria, P.J.G. Mulders, M. Radici, A. Signori, JHEP 11, 076 (2015), 1508.00402

[49] J.W. Qiu, M. Schlegel, W. Vogelsang, Phys. Rev. Lett. 107, 062001 (2011), 1103. 3861

[50] W.J. den Dunnen, J.P. Lansberg, C. Pisano, M. Schlegel, Phys. Rev. Lett. 112, 212001 (2014), 1401.7611

[51] A. Signori, Few Body Syst. 57, 651 (2016), 1602.03405

[52] J.P. Lansberg, C. Pisano, M. Schlegel, Nucl. Phys. B920, 192 (2017), 1702.00305

[53] D. Boer, S.J. Brodsky, P.J. Mulders, C. Pisano, Phys. Rev. Lett. 106, 132001 (2011), 1011.4225

[54] C. Pisano, D. Boer, S.J. Brodsky, M.G.A. Buffing, P.J. Mulders, JHEP 10, 024 (2013), 1307.3417 